\title{
Representación del cambio climático en YouTube: un análisis cuantitativo de los vídeos más populares
}

Recibido: 01/04/2019

Aprobado por pares: 31/10/2019
Enviado a pares: 06/06/2019

Aceptado: 09/10/2019

DOI: $10.5294 /$ pacla.2021.24.1.5

Para citar este artículo / to reference this article / para citar este artigo Muñoz-Pico, H. P., León, B. y García, A. N. (2021). Representación del cambio climático en YouTube: un análisis cuantitativo de los vídeos más populares. Palabra Clave, 24(1), e2415. https://doi.org/10.5294/pacla.2021.24.1.5

\section{Resumen}

Este artículo examina si los contenidos de YouTube sobre el cambio climático contribuyen a que se generen nuevas propuestas de comunicación, con voces y enfoques distintos, lo que implicaría una mejor información de los ciudadanos y podría facilitar su implicación y participación en la toma de medidas necesarias para enfrentar el problema (decisiones de consumo, presión a los políticos para que adopten medidas, etc.), o si, por el contrario, se han amplificado las voces que, en general, predominan en los medios, como las de los políticos. Mediante un análisis de contenido, este estudio analiza la representación del cambio climático en los vídeos más populares en YouTube. Tras examinar una muestra de 288 vídeos difundidos durante el periodo octubre 2016-octubre 2017, se constata que los políticos, como el expresidente estadounidense Donald Trump, son las voces más citadas

\footnotetext{
https://orcid.org/0000-0002-8280-5456. Universidad Internacional del Ecuador, Ecuador. himunozpi@uide.edu.ec https://orcid.org/0000-0001-8556-9367. Universidad de Navarra, España. bleon@unav.es

$\triangle$ https://orcid.org/0000-0002-4017-5791. Universidad de Navarra, España. albgarcia@unav.es
} 
en las piezas de YouTube sobre cambio climático, seguidas de periodistas y científicos. Respecto de los productores de esos vídeos, los medios de comunicación ocupan un lugar privilegiado de popularidad. Incluso, parte de sus contenidos se reutilizan total o parcialmente por otros usuarios de la plataforma. La vocación principal de los mensajes es informativa, con un discurso enfocado, sobre todo, en las consecuencias del cambio climático en los países desarrollados. Los resultados del análisis de contenido también ponen de manifiesto que las informaciones que ayudan a entender los procesos en los que se inscriben los problemas ambientales quedan relegadas por la prominencia de la coyuntura política en los vídeos más populares sobre el cambio climático en YouTube. Entre las conclusiones de este estudio, destaca el predominio de políticos, funcionarios o exfuncionarios por encima de voces expertas, como los científicos, que conlleva el riesgo de una menor discusión social e implicación ciudadana en las acciones necesarias para enfrentar el cambio climático.

\section{Palabras clave (Fuente: tesauro de la Unesco)}

Cambio climático; YouTube; ciencia politizada; periodismo; medio ambiente. 


\section{Climate Change Representation on YouTube: Quantitative Analysis of the Most Popular Videos}

\section{Abstract}

This article examines whether YouTube content on climate change allows for fresh communication initiatives with diverse voices and approaches, implying better information for citizens and involving them in taking the necessary steps to face the problem (consumption decisions, pushing politicians to adopt measures, among others), or amplifies the traditionally predominating voices in the media, such as politicians. This study explores climate change representation in the most popular videos on YouTube through content analysis. After reviewing a sample of 288 videos uploaded during October 2016-October 2017, we found that politicians, such as former US President Donald Trump, are the most quoted in YouTube videos on climate change, followed by journalists and scientists. Regarding video producers, the media hold a privileged popular position, as even other YouTubers reuse their contents totally or partially. Their messages intend to be informative with a speech focused on the consequences of climate change in developed countries. The content analysis results also show that, in the most popular YouTube videos on climate change, the information that helps to understand the processes implicated in environmental issues is pushed into the background by the prominence of the political situation. In conclusion, politicians, officials, or former officials prevail over expert voices, such as scientists, risking social debate and citizen participation in the actions to tackle climate change.

\section{Keywords (Source: Unesco Thesaurus)}

Climate change; YouTube; politicized science; journalism; environment. 


\section{Representação da mudança climática no YouTube: análise quantitativa dos vídeos mais populares}

\section{Resumo}

Neste artigo, é analisado se os conteúdos do YouTube sobre a mudança climática contribuem para que novas propostas de comunicação, com vozes e abordagens distintas, sejam geradas, o que implicaria uma melhor informação dos cidadãos e poderia facilitar seu envolvimento e participação na tomada de medidas necessárias para enfrentar o problema (decisões de consumo, pressão aos políticos para que adotem medidas, entre outras) ou se, ao contrário, são amplificadas as vozes que, em geral, predominam nos meios, como as dos políticos. Por meio de uma análise de conteúdo, neste estudo, é analisada a representação da mudança climática nos vídeos mais populares no YouTube. Após examinar uma amostra de 288 vídeos difundidos entre outubro de 2016 e outubro de 2017, é constatado que os políticos, como o ex-presidente Donald Trump, são as vozes mais citadas no YouTube sobre mudança climática, seguidas de jornalistas e cientistas. A respeito dos produtores desses vídeos, os meios de comunicação ocupam um lugar privilegiado de popularidade. Ainda, parte de seus conteúdos é reutilizada total ou parcialmente por outros usuários da plataforma. A intenção principal das mensagens é informativa, com um discurso focado, principalmente, nas consequências da mudança climática nos países desenvolvidos. Os resultados da análise de conteúdo também evidenciam que as informações que ajudam a entender os processos nos quais estão inseridos os problemas ambientais ficam abandonadas pela prominência da conjuntura política nos vídeos mais populares sobre a mudança climática no YouTube. Entre as conclusões deste estudo, destaca o predomínio de políticos, funcionários ou ex-funcionários por cima de vozes especializadas, como as dos cientistas, o que implica o risco de uma menor discussão social e envolvimento cidadão nas ações necessárias para enfrentar a mudança climática.

\section{Palavras-chave (Fonte: tesauro da Unesco)}

Mudança climática; YouTube; ciência politizada; jornalismo; meio ambiente. 


\section{Introducción}

Desde comienzos del siglo XXI han crecido notablemente los productores de contenido alternativos a los medios de comunicación tradicionales, que distribuyen sus contenidos en plataformas web como YouTube, la más popular para la diseminación de vídeos. Por eso, resulta de especial interés conocer qué contenidos generan sobre temas de relevancia social, económica y ambiental, como el cambio climático. Se pretende identificar las principales fuentes informativas utilizadas en los vídeos sobre el cambio climático más populares en YouTube, es decir, explorar quién y cómo contribuye a crear la agenda del cambio climático al margen de los medios tradicionales. En el contexto actual, esto es especialmente relevante dado que los medios de comunicación tradicionales han demostrado no ser una fuente de información solvente ni suficiente sobre el cambio climático. Los periodistas, por ejemplo, han entendido la norma periodística del equilibrio como la búsqueda de la opinión contraria al consenso científico (Boykoff y Boykoff, 2007).

Para ello, en primer lugar, trazamos un repaso teórico a la relación entre los medios de comunicación y el cambio climático, a partir de la base de que los medios son la principal fuente de información ciudadana sobre este. Después, cerramos el foco para explorar cómo, en lugar de los científicos, son los políticos las fuentes predominantes en la información sobre cambio climático y reflexionamos sobre el papel que YouTube y el contenido generado por los usuarios (user-generated content [UGC]) desempeñan en la transmisión de los valores en torno al cambio climático. Una vez concluido el repaso al estado de la cuestión, se detalla la metodología empleada en el análisis de contenido de los 288 vídeos que componen la muestra que estudiamos. Tras exponer los resultados de nuestra investigación, en la sección última se debaten, a la luz de los datos obtenidos, aspectos como el papel de los creadores originales de contenidos relacionados con el cambio climático, el problema de la tergiversación al replicar o reutilizar materiales y el predominio de los Estados Unidos como principal marcador de la agenda informativa con respecto a este. 


\section{El cambio climático en los medios de comunicación: una cobertura insuficiente}

En 2017, una parte de los reportes en medios estuvieron centrados en la denominada postura negacionista (la que refuta que el calentamiento global, de existir, sea debido principalmente a causas humanas) del expresidente estadounidense Donald Trump respecto del cambio climático y su influencia en naciones clave como China y la India. El pico más alto de la cobertura se dio en junio, cuando Trump anunció la retirada de su país del Acuerdo de París (Boykoff et al., 2018). Esto evidencia que, aunque de forma escasa el cambio climático es objeto de cobertura por parte de los medios de comunicación (Erviti, 2013; Fernández-Reyes et al., 2015; León, 2013), el tema se aborda desde ejes tan dispares y variados como su relación con el aumento del hambre (Informe21.com, 2018), la dificultad que genera en el despegue de los aviones y el aumento de las turbulencias (NYtimes.com, 2018) o, incluso, sobre cómo la pobreza genera nuevos refugiados del clima (Theguardian.com, 2018).

En este sentido, como parece lógico siguiendo los criterios de noticiabilidad, la difusión periodística en torno al cambio climático crece especialmente cuando se realizan eventos internacionales como la cumbre de París de 2015. De hecho, como han demostrado Barkemeyer et al. (2017, p. 1047), los eventos internacionales sobre el clima impactan significativamente la atención que los medios otorgan al problema ambiental. En su estudio, compararon la cobertura de 113 periódicos en 41 países y encontraron que, en la exposición de un país al cambio climático o sus esfuerzos de mitigación, existía una correlación entre cobertura periodística y el compromiso de dichos países con políticas medioambientales.

La cobertura periodística del cambio climático ha sido ampliamente investigada desde la academia, con resultados que cuestionan la labor de los medios. Por ejemplo, Pasquaré y Venturini (2017) analizaron el contenido de 171 artículos de noticias de dos periódicos digitales italianos durante 2014. El estudio se gestó a propósito de una anomalía climática que produjo "un año sin verano" (marcado por temperaturas frías, alta nubosi- 
dad, lluvia y tormentas eléctricas frecuentes y abundantes) y hallaron que tan solo una cantidad limitada de noticias insinuaba el papel del cambio climático en el problema. ${ }^{4}$ Por su parte, Fernández-Reyes (2016) comparó la cobertura de las cumbres de Copenhague (2009) y París (2015) en los diarios españoles El País, El Mundo y La Vanguardia, y descubrió que el encuentro de París recibió menos atención mediática que el de Copenhague. La razón, como ha apuntado Boykoff (2016), pudo deberse a la influencia del contexto: la primera cumbre se produjo semanas después del "Climategate" y las conversaciones entre los países desarrollados y en desarrollo proporcionaron mucho drama, mientras que en la segunda cumbre, la de Francia de 2015, se había creado un ambiente más confiado tras años de diplomacia. Por citar un último estudio relevante, Boykoff (2012) analizó artículos periodísticos y segmentos de televisión de medios estadounidenses durante la conferencia de Cancún en 2010. Entre sus hallazgos destaca que se discutieron las oportunidades económicas con más frecuencia que los impactos económicos y que tanto los activistas como las voces indígenas quedaron marginados de la cobertura periodística.

Si partimos de la base de que los medios de comunicación son la principal fuente de información ciudadana sobre el cambio climático (Brulle et al., 2012; Meira et al., 2013; Wilson, 2000) y de que hay una estrecha conexión entre la cobertura en prensa y el interés público (Fernández-Reyes et al., 2015), podemos afirmar que los medios de comunicación desempeñan un papel clave en la percepción social del cambio climático. Sin embargo, el tema tiene diversos ángulos que conviene considerar, que evidencian cierto abismo entre la preocupación reflejada por los medios y la exhibida por la opinión pública. Como han examinado Stokes et al. (2015), en China y los Estados Unidos, los dos mayores emisores de gases de efecto invernadero (GEI), solo el 18 y el $45 \%$ de personas, respectivamente, dicen que el cambio climático es un problema muy grave. Por comparación, la mediana mundial es del $54 \%$ (2015). Estos datos son alarmantes si consideramos, por ejemplo, que la población china mayor de 15 años ronda

4 Existen varios estudios (Herring et al., 2015; Nimbus, 2014) que dan cuenta de la relación de las anomalías climáticas producidas en 2014 en todo el planeta, incluida Italia, con el cambio climático. 
los 1079 millones de habitantes, lo que implica que el $82 \%$ que no considera el cambio climático como un problema "muy grave" supone más de 884 millones de personas. No obstante, aunque pueda sonar paradójico, la cuestión ambiental es también, incluso, ignorada por quienes padecen sus consecuencias. Como menciona Whitmarsh (2008, p. 368), las personas que han sido víctimas de inundaciones rara vez las asocian con el cambio climático y no están más preocupadas o propensas a tomar medidas para enfrentarlo que otros individuos.

La representación mediática del cambio climático ha dejado de lado muchas historias que ayudarían a entender los procesos en los que se inscribe el tema, sobre todo, por la coyuntura política. La prominencia de las noticias sobre el cambio climático asociadas a Trump en 2017, conocido como el fenómeno "Trump Dump", da cuenta de cómo la atención de los medios en las acciones del mandatario dejó de lado eventos y problemas de verdadera relevancia medioambiental. Trump fue nombrado 19184 veces en 4117 informaciones de The Washington Post, The Wall Street Journal, The New York Times, USA Today y Los Angeles Times. Es decir, un promedio de 4,7 veces por artículo (Boykoff et al., 2018). Esta amplia representación de Trump en la prensa que informa del cambio climático es relevante, puesto que pone de manifiesto que, muchas veces, las voces expertas en el cambio climático se pierden en las coberturas, lo que puede traducirse en una menor discusión social y menos conciencia pública.

Además, Gurwitt et al. (2017) revelaron cómo la mayoría de la cobertura de la Conferencia sobre el Clima de París (COP21) dejó a los lectores con poca comprensión de los temas de alta prioridad para las naciones en desarrollo. Por ejemplo, hubo poca cobertura sobre la adaptación al cambio climático, que busca reducir la vulnerabilidad ante los efectos del cambio climático, y una mucho mayor de la mitigación, que se enfoca en reducir y limitar las emisiones de GEI. Además, los artículos subestimaron cuestiones clave para las naciones más pobres, como la equidad y los derechos humanos. Las historias en muchos de los casos eran actualizaciones sobre las conversaciones o se centraron en los activistas, las acciones de los líderes mundiales en la conferencia y los efectos ambientales del cambio climático. 
Esta tendencia a mostrar noticias sesgadas hacia los países desarrollados, con poca discusión de los países más vulnerables o los problemas importantes para ellos, tiene varias explicaciones, como el contexto y el atractivo mediático al que hemos hecho referencia, pero también que los emporios mediáticos más grandes están en los países desarrollados como los Estados Unidos, donde las empresas han invertido más para ampliar su alcance en el extranjero (Zenith, 2017).

El tema se agrava con ciertas rutinas periodísticas que llevan a una cobertura deficiente de los medios. Boykoff y Boykoff (2004) encontraron que la cobertura del clima contempla normas como el equilibrio. En general, los periodistas suelen contrastar la información con la inclusión de puntos de vista que en el cambio climático es el de científicos que muestran algún grado de escepticismo sobre este y de los ya mencionados negacionistas. Como han explicado Maibach et al. (2010), esta tendencia periodística hacia el equilibrio epistemológico puede adulterar la noticia, puesto que, en ocasiones, no transmite bien que el consenso científico en torno al hombre como causante del cambio climático es del $97 \%$ (2014). Erviti (2013) agrega que la información relacionada con el cambio climático tiene que cumplir criterios de noticiabilidad que difícilmente ostenta el problema ambiental, como la actualidad y la novedad. Esto resulta más agudo en la televisión que exhibe una fuerte orientación hacia el espectáculo. Por eso, el "gancho" noticioso suelen ser las cumbres climáticas o la publicación de nuevos datos, primando lo urgente e impactante sobre lo importante.

En consideración a lo anterior, no sorprende que la principal voz del cambio climático sea la de los políticos que cuentan con amplios equipos de comunicación pública y no la de los científicos que, además, deben lidiar con la dificultad de producir discursos más cercanos para el entendimiento del público en general.

\section{YouTube y la posibilidad de nuevos actores informativos}

En un entorno mediático donde los políticos y, en menor medida, los activistas copan las noticias relacionadas con el cambio climático (Mason, 2011; 
Olesen, 2015), internet ha servido para que varias de las voces interesadas en el cambio climático puedan hacerse escuchar y distribuir sus contenidos sin necesidad de intermediarios, como los medios de comunicación. En YouTube, por ejemplo, el sitio con más visitas en el mundo después de Google (Alexa, 2018), se visualizan diariamente más de mil millones de horas de contenidos. Por eso, se pone el foco en YouTube, porque es el principal repositorio de vídeo online: a diario genera más de 1000 millones de horas de reproducciones (YouTube, 2019) y se estimaba que para 2020 llegaría a tener una presencia mensual equivalente a cinco millones de años (Cisco, 2017). Además, el vídeo es una de las herramientas más importantes del nuevo ecosistema digital. Se estima que a escala mundial tres billones de minutos de contenido audiovisual cruzarán internet cada mes para 2022. Eso es 1,1 millones de minutos de vídeo transmitidos o descargados por segundo (Cisco, 2019). Además de la gama de opciones que ofrece YouTube, ciencia y tecnología resultó ser significativo entre los contenidos más populares de la plataforma a nivel mundial, situado en el segundo lugar solo por detrás de entretenimiento (Erviti y León, 2014).

Hay pocos estudios previos que analizan las fuentes en los vídeos de cambio climático publicados en YouTube. Salazar y Barroga-Jamias (2014) examinaron el contenido de 95 vídeos sobre la biodiversidad filipina y encontraron que alrededor del $80 \%$ de las fuentes citadas eran individuos y empresas privadas. Aunque en ese país la biodiversidad es una de las principales preocupaciones ambientales, el tema predominante de los vídeos fue la belleza ambiental. Por su parte, Erviti (2018, p. 33) buscó el término "cambio climático" en la sección de vídeos de Google y, a partir de una muestra de 300 piezas, encontró que un número apreciable de científicos (93) aparecía en los vídeos de cambio climático; las instituciones científicas son las que más los incluyen como fuentes de información.

YouTube es la muestra de que el contenido generado por usuarios puede llegar a ser tan popular como el profesional. Sin embargo, cabe preguntarse si los contenidos subidos en plataformas como esa han contribuido a que se generen nuevas propuestas para la comunicación del cambio climático o si, por el contrario, se han amplificado las voces que en general aparecen en los medios, como las de los políticos y, en menor medida, los activistas. 


\section{Metodología}

Dado el rápido crecimiento del vídeo online (este estudio pretende identificar, mediante el análisis de contenido, cuáles son las fuentes y voces del cambio climático en los vídeos más populares en YouTube), se entenderá por vídeo popular todo contenido cuya difusión crece exponencialmente debido al control del usuario sobre la distribución. Este proceso dura un tiempo limitado en el cual se multiplican las visualizaciones, incluso hasta miles de millones. Hay que considerar que el contenido, cuando se comparte, pierde su condición estática (de ubicación, de fondo o ambas) y se constituye en un recurso cultural que sirve para comunicar. Por ello, para la muestra de este estudio, se tomaron en consideración los vídeos de más de 1000 visualizaciones, cuyo proceso de crecimiento era proporcional al alcanzado, ya que los procesos de crecimiento que son proporcionales al tamaño alcanzado se prestan al crecimiento exponencial, como ya estudió Dawkins (1994, pp. 396-397) al referirse al concepto de viralidad. Así, aunque se eligió el año que discurre desde octubre de 2016 hasta octubre de 2017, en los resultados había vídeos que apenas contaban con una antigüedad de una hora o de un día, pero que ya habían alcanzado las 1000 visualizaciones. Como se ha apuntado antes, se escogió esta plataforma por su amplio posicionamiento en internet, mucho más popular y omnipresente que cualquiera de las alternativas (Vimeo, Dailymotion, Metacafe, etc.).

Para examinar el mensaje de cada vídeo, se plantearon las siguientes preguntas de investigación:

P1. ¿Quiénes son los productores de los vídeos más vistos sobre cambio climático en YouTube?

En esta pregunta, nos interesa conocer si se trata de medios de comunicación que replican su propio contenido en YouTube o si, por el contrario, hay nuevos actores (gobiernos, instituciones públicas, ONG o, incluso, particulares) que se aventuran en la producción y distribución del vídeo online para influir en la agenda sobre el cambio climático.

P2. ¿Cuáles son las fuentes citadas con mayor frecuencia? 
En un entorno, el científico, donde la autoridad epistemológica es relevante para transmitir rigor y confianza en el mensaje, esta pregunta permite evaluar si las fuentes a las que más recurren los vídeos son, en efecto, científicos, políticos, periodistas especializados, activistas, celebridades, etc.

P3. ¿Cuál es el escenario principal de la información?

Aquí se estudia el lugar donde se desarrolla principalmente la información. Así, se establecieron los siguientes escenarios: global, Europa, América, Asia, África, Oceanía, Ártico, Antártico o un hábitat específico. ${ }^{5} \mathrm{Se}$ resolvió elegir continentes como escenarios principales para tener resultados uniformes, ya que la comparación entre países podría resultar asimétrica, por ejemplo, si hablamos de países desarrollados o en vías de desarrollo.

P4. ¿Cuál es la vocación principal de los mensajes?

Para esta pregunta, el estudio estableció los siguientes parámetros:

- Denuncia. Aviso sobre el estado ilegal, irregular o inconveniente de hechos relacionados con el cambio climático.

- Informativa. Da noticia de hechos relacionados con el cambio climático, de una forma aséptica, que aspira a resultar imparcial o neutral.

- Crítica. Se inclina a enjuiciar hechos y conductas en general de forma desfavorable.

- Didáctica. Tiene como finalidad fundamental enseñar o instruir sobre el cambio climático.

- Implicación social. Su objetivo es comprometer a la gente en la lucha contra el cambio climático.

- Burla. Procura poner en ridículo a alguien o algo. Su rechazo del cambio climático es mordaz, poco serio. Se niega a participar en el debate y se caracteriza por sus reflexiones satíricas.

5 Según el Diccionario de la lengua española (Real Academia Española [RAE], 2014), el hábitat es el lugar de condiciones apropiadas para que viva un organismo, especie o comunidad animal o vegetal. Existen hábitats terrestres, como los bosques y los desiertos; y acuáticos, como los océanos y los lagos. 
- Otro. En esta categoría entraría cualquier variante no recogida en las seis anteriores.

P5. ¿Se menciona el carácter antropogénico del cambio climático?

Conocer si existe una atribución directa o indirecta de que las actividades humanas inciden en el cambio climático sirve para determinar si los contenidos propician o no la generación de una verdadera conciencia ambiental. Es decir, ante esta pregunta la respuesta es binaria: sí o no.

P6. ¿Cuál es encuadre principal de los vídeos?

Para esta pregunta, se establecieron los siguientes parámetros:

- Pesimista no pragmático. La situación actual y sus perspectivas futuras se presentan en forma alarmista y fuera del control humano. Se emplea un lenguaje extremista, con un tono de urgencia y desesperación ante los efectos acumulativos y la irreversibilidad de la degradación. Del contenido se deriva la idea de que el problema es de tal magnitud que, prácticamente, no tiene sentido alguno intervenir, lo que produce parálisis y resignación.

- Pesimista pragmático. Aunque las perspectivas futuras se presentan en forma alarmista, considera que las actuaciones individuales, como ahorrar energía en el hogar, o las gubernamentales podrían mermar los efectos catastróficos del cambio climático.

- Optimista no pragmático. Supone que todo está bien y que el problema no es tan grave. Rechaza y se burla de los alegatos alarmistas y con ello del propio cambio climático. Al rechazarlo, se opone a participar en el debate y parece inmune a los argumentos científicos.

- $\quad$ Optimista pragmático. Supone que todo estará bien si hacemos algo. Consiste en solicitar al mayor número de personas emprender pequeñas actuaciones para contrarrestar el cambio climático considerando un efecto sinérgico. La sumatoria de acciones individuales produce un impacto no desdeñable. El lenguaje empleado es el de la facilidad, la 
comodidad y la simplicidad del esfuerzo en la vida doméstica. Se ve en referencia a los focos ahorradores, los coches eléctricos, etc.

- Otro. En esta categoría entraría cualquier variante no recogida en las cuatro anteriores.

Los datos obtenidos para estas seis preguntas estuvieron sujetos a ciertos condicionantes metodológicos. Para el análisis, se escogieron los vídeos más vistos, entre octubre de 2016 y octubre de 2017, al buscar "climate change”, en consideración a las siguientes características y filtros:

- Fecha de publicación. Se seleccionaron los vídeos publicados en ese año para que no existan diferencias significativas en relación con su antigüedad.

- Tipo. Entre las opciones ofrecidas por la plataforma se escogió "vídeos". De lo contrario, la búsqueda arroja resultados tales como "canales" y las "listas de reproducción" que afecta a las estadísticas.

- Duración. Corta, de menos de cuatro minutos. Como es lógico, existen piezas sobre el cambio climático de hasta más de una hora de duración. Sin embargo, acotar el tiempo a cuatro minutos está avalado por tres razones. La primera es que este filtro en la duración permite que la prueba intercodificadores no quede afectada, algo que sí ocurriría con contenidos muy extensos, ya que los vídeos para el análisis deben ser revisados varias veces para verificar que no se excluyeron o malinterpretaron variables. La segunda es que la razón de restringir la muestra parte también de que el YouTube solo permite dos filtros en este sentido: vídeos cortos de menos de cuatro minutos y vídeos largos mayores de veinte minutos. Y, por último, que los resultados no se vieran afectados por comparar contenidos muy distintos, por ejemplo, una noticia con un documental, ya que la idea de este estudio es comparar contenidos similares en edad, tipo, duración, etc.

- Ordenar por número de visualizaciones.

Antes de aplicar estos filtros, se eliminaron todas las cookies del ordenador y se limpió la memoria caché. Asimismo, la pestaña para la búsqueda 
en YouTube se abrió en modo privado para evitar que la aplicación utilice el historial del ordenador para desplegar sus resultados.

La búsqueda inicial arrojó 4390000 resultados. Luego de los filtros aplicados quedaron 3940 000. De esa cifra, la plataforma solo mostró 597 vídeos, repartidos en 30 pestañas. Para la selección de la muestra, se escogieron los vídeos con 1000 o más visualizaciones (313) y tras la revisión final se excluyeron 11 piezas que eran clones, ocho que habían sido eliminados por los administradores de las cuentas durante el análisis, cuatro que trataban temas distintos del cambio climático y dos que estaban en ruso y filipino (todo el resto estaba en inglés, la actual lingua franca en el mundo, por lo que, de hecho, el estudio solo analiza vídeos en la lengua de Shakespeare). En consecuencia, la muestra final quedó en 288 vídeos, que fueron codificados por dos codificadores independientes.

El acuerdo intercodificadores se midió mediante la $\kappa$ de Cohen, una prueba comúnmente usada para determinar el acuerdo entre el juicio de dos codificadores cuando hay variables categóricas. Los datos fueron procesados en el software estadístico SPSS y estos fueron los resultados:

[P1] La $\kappa$ de Cohen de la prueba intercodificadores sobre el "Tipo de productor" arrojó el siguiente resultado, considerado como óptimo, $\kappa=0,903$, IC $95 \%[0,772,1,034], \mathrm{p}<0,001$.

[P2] En cuanto a la identidad de las fuentes, los resultados $\kappa$ quedaron como se detalla en la tabla 1.

\section{Tabla 1. Identidad de las fuentes}

\begin{tabular}{|c|c|c|}
\hline Identidad de las fuentes & Kappa & $\mathbf{9 5} \%$ IC \\
\hline Científico, académico & 0,889 & {$[0,675,1,103]$} \\
\hline Funcionario o exfuncionario & 0,864 & {$[0,682,1,046]$} \\
\hline Periodista, presentador, comentarista, comunicador & 0,783 & {$[0,410,1,193]$} \\
\hline Empresario & 1,000 & {$[1,000]$} \\
\hline Economista & $*$ & $*$ \\
\hline Politólogo & $*$ & $*$ \\
\hline
\end{tabular}




\begin{tabular}{|c|c|c|}
\hline Identidad de las fuentes & Kappa & 95 \% IC \\
\hline $\begin{array}{c}\text { Representante de asociación en defensa del medio ambiente, ambientalista, } \\
\text { ecologista }\end{array}$ & 0,902 & {$[0,714,1,090]$} \\
\hline Famoso & 0,911 & {$[0,740,1,082]$} \\
\hline Representante de organismo internacional o de ONG & 1,000 & $*$ \\
\hline Representante religioso & $*$ & $* 1,000]$ \\
\hline Médico, profesional de salud & $*$ & $* 1,000]$ \\
\hline Ciudadano corriente & 1000 & $*$ \\
\hline Otro & $*$ & $*$ \\
\hline
\end{tabular}

* Sin estadísticos porque la codificación de C1 y C2 fueron iguales.

Fuente: elaboración propia.

En la segunda unidad de análisis "Identidad de las fuentes", los codificadores debieron marcar la aparición de cada fuente en los vídeos y el número de veces que lo hacían. Tal como se muestra en la tabla 1 , hubo un acuerdo muy bueno entre los juicios de los dos codificadores al anotar la aparición de "Científico, académico", "Funcionario o exfuncionario", "Empresario", "Economista”, "Politólogo”, "Representante de asociación en defensa del medio ambiente, ambientalista, ecologista”, "Famoso", "Representante de organismo internacional o de ONG", "Representante religioso", "Médico, profesional de salud", "Ciudadano corriente", "Otro", así como un buen acuerdo al apuntar la aparición de "Periodista, presentador, comentarista, comunicador".

[P3] La prueba intercodificadores $\kappa$ de Cohen arrojó un juicio perfecto en "Escenario principal de la información", $\kappa=1,000$, IC $95 \%[1,000]$, $\mathrm{p}<0,001$.

[P4] La $\kappa$ de Cohen para "Vocación principal del mensaje" arrojó un acuerdo muy bueno entre los juicios de los codificadores, $\kappa=0,957$, IC $95 \%[0,875,1,039], \mathrm{p}<0,001$.

[P5] La $\kappa$ de Cohen para "Mención del carácter antropogénico del cambio climático" arrojó un acuerdo muy bueno entre los juicios de los dos codificadores, $\kappa=1,000$, IC $95 \%[1,000], \mathrm{p}<0,001$. 
[P6] La $\kappa$ de Cohen para "Encuadre temático principal de los vídeos" arrojó un acuerdo muy bueno entre los juicios de los dos codificadores, $\kappa=1,000$, IC $95 \%[1,000], p<0,001$.

Dado que el análisis de contenido es una técnica de constatación de frecuencias (López-Noguero, 2002), hemos optado por presentar los resultados de las preguntas en gráficos circulares para que el lector pueda apreciar las proporciones de las variables analizadas en cada categoría. Además, en la primera pregunta, se utiliza también un gráfico de columnas agrupadas para comparar la presencia de actores de interés (fuentes) en los contenidos emitidos por medios de comunicación.

\section{Resultados}

El $53 \%$ de los vídeos más populares analizados (154) sobre cambio climático difundidos en YouTube fueron producidos por medios de comunicación. Un $23 \%$ (66) correspondió a "Otros", donde se encuentran empresas, organizaciones religiosas, partidos políticos y otras instituciones que tienen una programación recurrente sobre diversos temas, incluido el ambiental (figura 1). De ellos, el 21,7\%, que representan cinco casos, tomaron sus contenidos de canales de televisión. Además, 17 de los 30 usuarios particulares que difundieron contenidos también los tomaron de medios de comunicación ( 16 de canales de TV y uno de radio).

\section{Figura 1. Tipo de productor.}

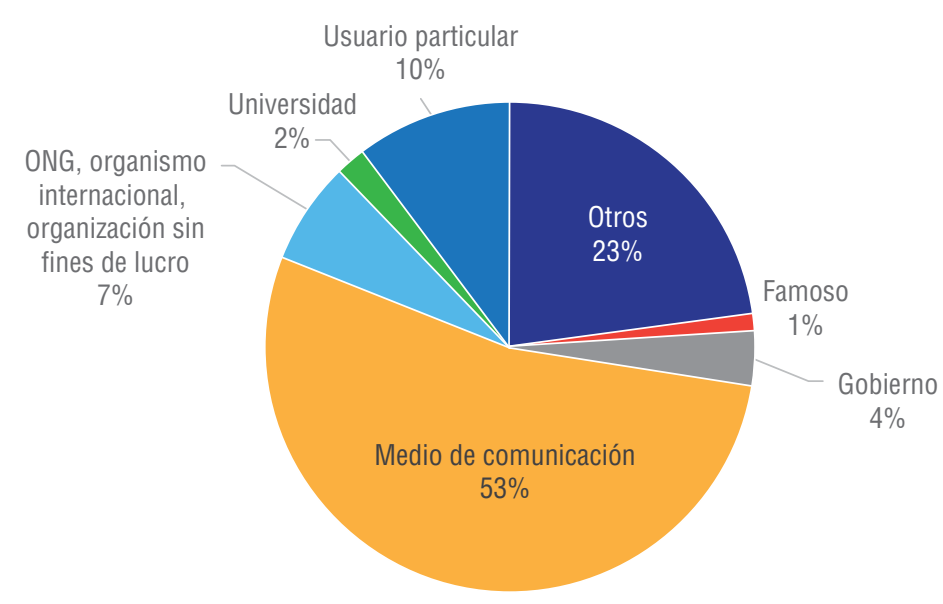

Fuente: elaboración propia. 


\section{Resultados [P1] ¿Quiénes son los productores de los vídeos más vistos sobre cambio climático en YouTube?}

En resumen, de los 288 vídeos más populares que conformaron la muestra, $176(61,11 \%)$ fueron contenidos que se emitieron previamente en medios de comunicación, especialmente televisión.

Dado que existen fuentes como las universidades, los gobiernos y las ONG que cuentan con departamentos de prensa, nos pareció interesante establecer cuántos de ellos estuvieron presentes en la producción de los medios. Se trata, por tanto, de cruzar dos variables: la del tipo de productor (en este caso, tomamos los producidos por medios de comunicación) y la de las fuentes citadas. Como se aprecia en la figura 2, estos fueron los resultados: 84 funcionarios o exfuncionarios (65\%), 38 científicos o académicos (30\%) y seis representantes de organismos internacionales u ONG (5\%).

\section{Figura 2. Fuentes citadas por los medios.}

Funcionarios o exfuncionarios públicos

Cinetíficos 0 académicos

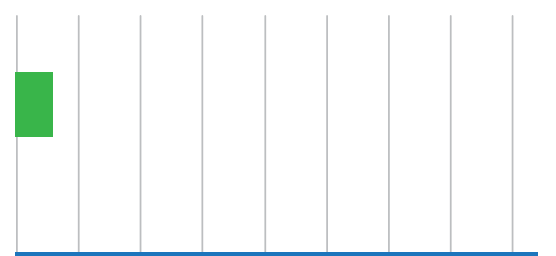

Representantes de organismos internacionales u ONG

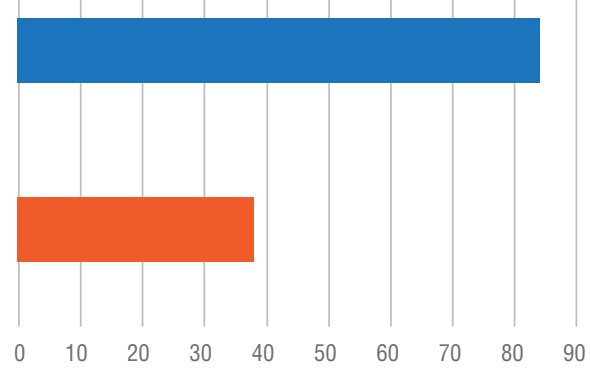

Fuente: elaboración propia.

\section{Resultados [P2] ¿Cuáles son las principales fuentes citadas?}

A diferencia de la figura 2 , a continuación volvemos a tomar la totalidad de los vídeos de nuestra muestra bajo una única variable: la identidad de las fuentes. Como se aprecia en la figura 3, en la muestra analizada se citó con mayor frecuencia a políticos, funcionarios o exfuncionarios (126 veces). Dada la fecha de toma de la muestra, el tema más destacado fue la de- 
cisión de los Estados Unidos de retirarse del Acuerdo de París, por lo que el expresidente Donald Trump y su equipo de ministros y asesores fueron constantemente mencionados. Luego, estuvieron los periodistas de medios de comunicación (73). En los informativos estadounidenses, es frecuente que se invite a un periodista especializado de un medio impreso a dar una entrevista, siendo este la única fuente en varios de los vídeos. En tercer lugar, se ubicaron los científicos o académicos (70).

\section{Figura 3. Identidad de las fuentes.}

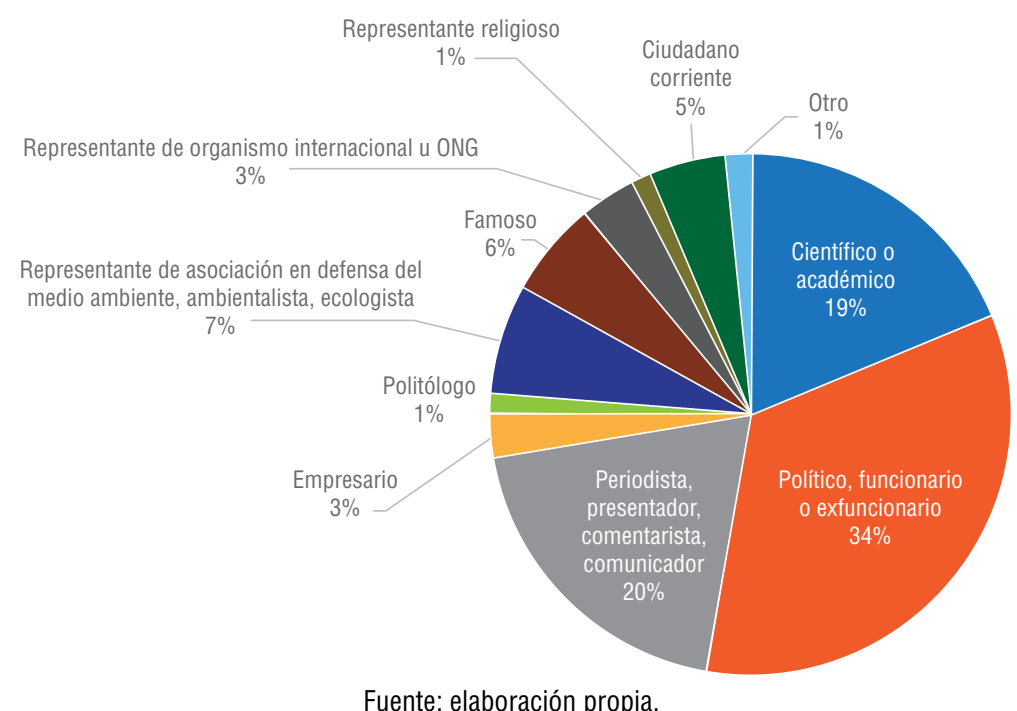

\section{Resultados [P3] ¿Cuál es el escenario principal de la información?}

Como era de esperar, dada la gran cobertura sobre las acciones y declaraciones del expresidente Donald Trump, el escenario principal de los vídeos de cambio climático analizados fue Norteamérica. Este resultado también pone de manifiesto la gran influencia en YouTube de la información generada allí.

Asia apenas fue el escenario del $2 \%$ de los vídeos, a pesar de tener uno de los principales países emisores de GEI (China). Este resultado, sin embargo, se ve influenciado por la búsqueda del término "cambio climático" en inglés y no en chino mandarín, su idioma oficial. Resultan significativos los datos del Ártico y la Antártida, con un 1 y un $3 \%$, respectivamente, que 
en la actualidad sufren el deshielo de sus glaciares a causa del cambio climático; Oceanía ( $4 \%$ ), que tiene países como Fiyi en crisis ambiental por los constantes azotes de ciclones; y África (4\%), un continente muy vulnerable al cambio climático, sobre todo por la sequía y el hambre (figura 4).

\section{Figura 4. Escenario principal de la información.}

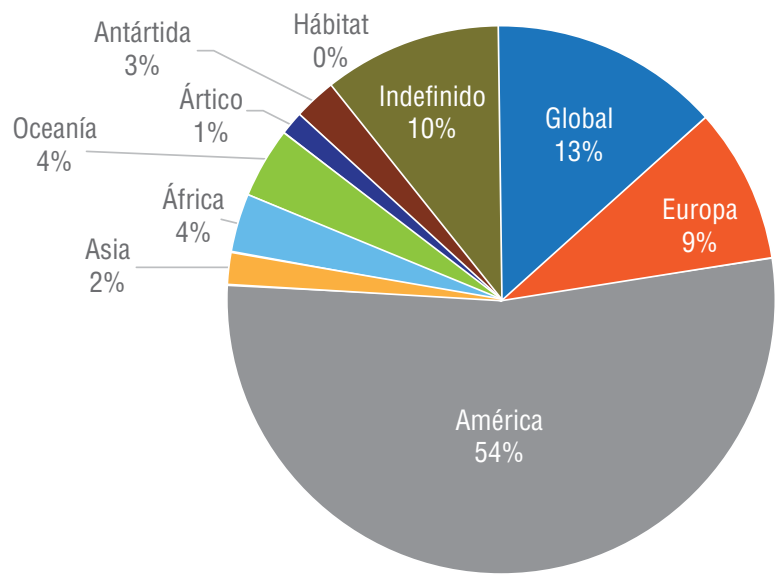

Fuente: elaboración propia.

\section{Resultados [P4] ¿Cuál es la vocación principal de los mensajes?}

La vocación principal de los mensajes fue "Informativa" (figura 5). Los denominados negacionistas también estuvieron representados en el segmento "Burla", donde se ubicaron los contenidos cuyo rechazo al cambio climático fue mordaz y poco serio. Por ejemplo, en el vídeo "Al Gore Loves This Climate Change Line About the Book of Revelation|SUPERcuts! \#509", el exvicepresidente estadounidense $\mathrm{Al}$ Gore aparece 11 veces, en distintos espacios televisivos, repitiendo que "cada noche las noticias son como una caminata por la naturaleza a través del Libro de la Revelación", y en la parte final se lo ve en la serie animada South Park donde habla de la existencia del hombre-oso-cerdo. ${ }^{6}$

6 En el episodio "ManBearPig" (10.6.), Al Gore asiste a la escuela de South Park para revelar un tema "muy serio": la existencia del hombre-oso-cerdo. La criatura es una alusión al cambio climático. El exvicepresidente aparece como un hombre perturbado y se lo ridiculiza varias veces. Llora porque nadie le cree y, en lugar de decir que quiere que lo tomen en "serio", dice "cereal". Incluso, se disfraza del monstruo que afirma haber visto. 


\section{Figura 5. Vocación principal del mensaje.}

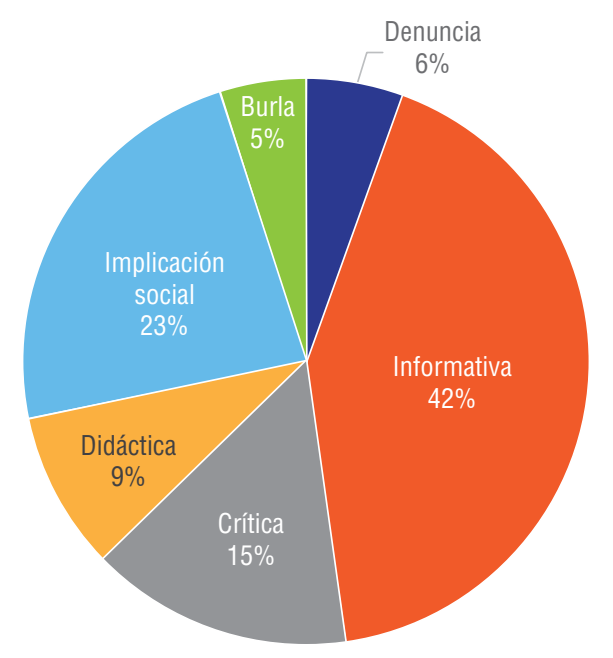

Fuente: elaboración propia.

\section{Resultados [P5] ¿Se menciona el carácter antropogénico del cambio climático?}

En esta ocasión, la pregunta implicaba una simplificación de la respuesta: un binario sí o no. Se buscaba comprobar hasta qué punto uno de los aspectos clave en el cambio climático (el papel del hombre como causante) quedaba reflejado en la muestra. Los resultados arrojan que solo en 71 de los 288 vídeos más populares que conformaron la muestra se atribuyó el carácter antropogénico del cambio climático. En los otros 217, no se mencionó al ser humano como responsable directo o indirecto del cambio climático (figura 6).

\section{Resultados [P6] ¿Cuál es encuadre principal de los vídeos?}

El encuadre principal de los vídeos fue "Pesimista pragmático" (220 casos). El lenguaje catastrofista de esos vídeos estuvo acompañado de imágenes impactantes asociadas a subidas en el nivel del mar, deshielo y fenómenos climáticos extremos, etc. A pesar de que se hicieron llamados a la acción, el problema se mostró tan ubicuo e inmenso que da la impresión de que no tiene sentido alguno intervenir. Por contraparte, 13 vídeos mostraron un encuadre optimista pragmático, más cercano a los ciudadanos, donde, incluso, se les enseña la forma de reducir su huella ecológica (figura 7). 


\section{Figura 6. Mención del carácter antropogénico del cambio climático.}

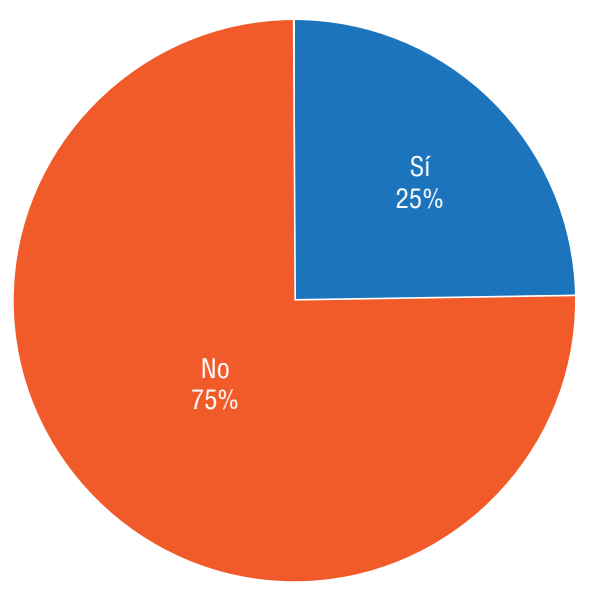

Fuente: elaboración propia.

\section{Figura 7. Encuadre principal de los vídeos.}

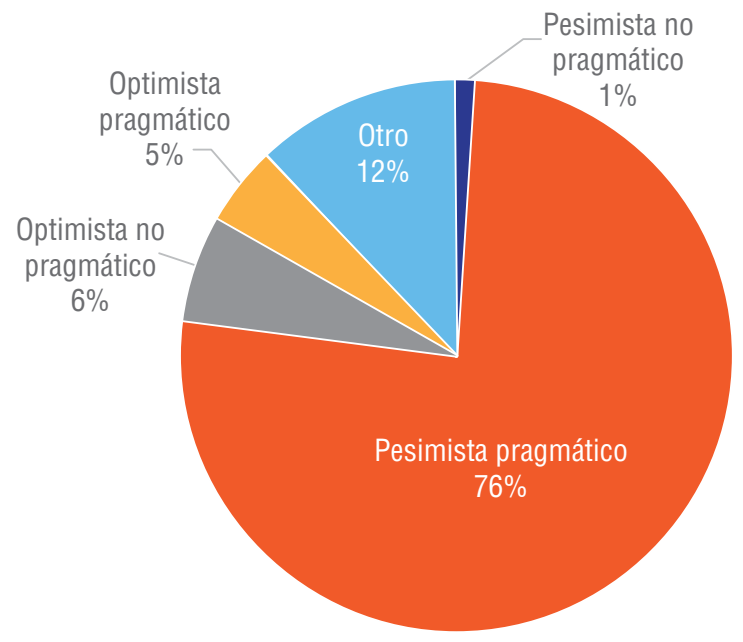

Fuente: elaboración propia.

\section{Discusión y conclusiones}

$\mathrm{Al}$ discutir los datos obtenidos, se aprecia la preeminencia de los medios tradicionales al marcar la agenda informativa, puesto que el $53 \%$ de los vídeos más populares en YouTube provienen de televisiones y periódicos. En este sentido, gobiernos, ONG y universidades aún deben redoblar esfuer- 
zos para comunicar sus investigaciones sobre cambio climático, al margen de los media tradicionales. En este estudio, aparecieron como productores en un porcentaje mínimo, a pesar de contar con temas relevantes, tales como el descubrimiento (por parte de las universidades de Brest y Waikato, así como por el Museo Australiano del Sur) de nuevas especies durante una investigación sobre el impacto del cambio climático en la vida marina de la Antártida. El hecho fue dado a conocer por el canal Al Jazeera en uno de los vídeos de mayor popularidad de la muestra revisada.

Los gobiernos y los famosos aparecieron como productores también en un porcentaje pequeño. En el primer caso, si nos fijamos en los Estados Unidos, el resultado se relaciona con el rechazo del Gobierno por entrar a una discusión seria sobre la crisis medioambiental. Por otra parte, aunque la capacidad para atraer audiencias de los famosos es evidente (como el rapero Prince Ea que estuvo dentro del top 20 de los vídeos más populares con una pieza donde les enseña a sus fanáticos cuatro maneras de combatir el cambio climático), el encuadre de sus mensajes no siempre se antoja como el más adecuado. Beyoncé, por ejemplo, apareció en un vídeo catastrofista y desalentador sobre el cambio climático. Esto supone un problema a la hora de crear conciencia: muchas veces la gente entra en negación no porque un tema le parezca irrelevante, sino porque siente que no puede actuar sobre él. En el caso del cambio climático, los encuadres pesimistas pueden acentuar esa actitud.

Llama la atención que no haya aparecido ningún médico o profesional de la salud en ninguno de los vídeos populares, sobre todo, porque, como han indicado varios investigadores (Maibach et al., 2010, Nisbet, 2009; Nisbet y Scheufele, 2009), el enfoque del cambio climático como un problema de salud pública es más efectivo en la participación de la audiencia que los marcos ambientales o políticos que actualmente dominan el discurso público. A los médicos les falta aumentar sus esfuerzos por visibilizar los efectos del cambio climático en la salud (Bauchner y Fontanarosa, 2014), ya que actualmente sigue siendo complicado relacionar ciertas enfermedades respiratorias, cardiovasculares o neumológicas con el cambio climático por la falta de una base de conocimientos sólida (Huerta, 2017). 
El uso de los contenidos de los medios en varios de los vídeos publicados por productores ajenos a estos plantea cuestiones relacionadas con la veracidad y el statu quo. El contenido que pasa de un lugar a otro, como de la televisión a YouTube, corre el riesgo de ser tergiversado, más aún si se consideran los intereses de determinados sectores, por ejemplo, el de empresas, cuyas actividades impactan significativamente el incremento de GEI, como las automovilísticas, de bebidas gaseosas y las que fabrican equipos domésticos que se incluyen entre los patrocinadores de las cumbres climáticas como la COP21. ${ }^{7}$ Por otra parte, replicar los contenidos de los medios puede desembocar en que se amplifiquen las voces de personas que no necesariamente son las más expertas y con mayor conciencia medioambiental, y se mantenga la inacción de ciertos sectores, incluidos los gubernamentales, para enfrentar el problema. Además, tal como sugieren Boykoff y Boykoff (2007), hay que recordar que la adherencia constante a las normas periodísticas en los medios, como el equilibrio y la novedad, llevan a una cobertura informativa deficiente sobre el cambio climático.

Que Norteamérica haya sido el escenario principal de la información y los funcionarios o exfuncionarios las fuentes principales, así como que la vocación principal de los mensajes fuera informativa, dan cuenta de la correspondencia de la agenda mediática con la política. ¿Cuál es el problema de que los políticos sean quienes guíen la agenda informativa? Que existen discursos populistas que pueden llegar a ser mucho más convincentes que los datos y crear juicios errados sobre el problema ambiental. Además, los enfoques informativos del cambio climático muchas veces se limitan a dar cuenta de hechos puntuales, como la declaración de un funcionario, y no comprometen a la gente en la lucha contra este. En este estudio, la vocación de implicación social estuvo detrás de la informativa, pero representó apenas la mitad de ella.

Norteamérica estuvo fundamentalmente representada por los Estados Unidos, un país desarrollado y con una vulnerabilidad distinta que otros países de ese continente, como los del sur, donde con los años se re-

7 La cumbre tuvo 34 patrocinadores. El listado completo se encuentra disponible en http://www.cop21paris.org/ sponsors-and-partners/sponsors 
crudecen fenómenos climáticos como El Niño y La Niña, y que necesitan un discurso enfocado, sobre todo, en la adaptación al cambio climático más que en la mitigación, ya que los principales emisores de GEI son los países industrializados.

El resto de los continentes apareció en un porcentaje individual inferior al $10 \%$. Esto llama la atención, ya que la búsqueda del término "climate change" respondió, entre otras cuestiones, a que el inglés es la lengua materna de más de 360 millones de personas en el mundo y la de otros 500 millones que lo hablan como segundo idioma (Babbel, 2016). Solo en África hay más países que hablan inglés que en Norteamérica. El hecho de que Norteamérica sea el escenario principal de la información pone en evidencia la interconexión planteada en estudios anteriores (Brulle et al., 2012; Boykoffy Roberts, 2007; Weingart et al., 2000) entre la cobertura mediática del cambio climático y la política, en los que se sugiere que los pronunciamientos sobre temas que se relacionan con la seguridad y el bienestar de la población (como el riesgo de una catástrofe global por el retiro de los Estados Unidos del Acuerdo de París) generan una importancia política que tiene un alto valor noticioso para los medios de comunicación. En este caso de la predominancia de Norteamérica como escenario de los vídeos, el anuncio de Trump de desvincular a los Estados Unidos del Acuerdo de París generó diversas opiniones en los funcionarios del ejecutivo y el legislativo de ese país que acapararon la atención de los medios de comunicación.

En cuanto a la vocación principal de los mensajes, resulta lógico el alto porcentaje de vocación "Informativa” (43\%), dado que los contenidos difundidos por los medios corresponden en su mayoría a la coyuntura noticiosa. En la muestra analizada, la decisión del mandatario estadounidense de retirar a su país del Acuerdo de París y la reacción de naciones como Francia y Alemania, así como de analistas en medios de comunicación, explica el alto número de noticias en torno al cambio climático. En segundo lugar, estuvo la vocación "Implicación social”, desprendida, en gran parte, del encuadre pesimista pragmático del cambio climático en el que se yuxtaponen los dos discursos citados como predominantes en el repertorio del cambio climático por Ereaut y Segnit (2006): el alarmista y el optimista pragmá- 
tico, en que, a pesar de que las perspectivas futuras se presentan en forma apocalíptica, se considera que las actuaciones individuales, como ahorrar energía en el hogar, o gubernamentales, enfocadas en reducir las emisiones de carbono, podrían mermar los efectos catastróficos del cambio climático.

En la mayoría de los vídeos, tampoco se alude al carácter antropogénico del cambio climático. Este aspecto resulta una limitante en la creación de conciencia sobre el problema: para actuar sobre el cambio climático, las personas deben saber que con algunas de sus acciones también afectan al planeta y que no es solo un tema de los gobiernos. Por tanto, las nuevas posibilidades de una plataforma como YouTube no están logrando cambiar sustancialmente el paradigma informativo en un aspecto tan relevante para combatir el papel del hombre en el cambio climático.

Finalmente, el encuadre pesimista pragmático se impuso por mucho sobre el resto de los encuadres (pesimista no pragmático, optimista pragmático y optimista no pragmático). La presentación casi apocalíptica del futuro, si bien capta la atención, no parece la más adecuada para motivar a la acción y al cambio de actitud. Retratar el cambio climático como un problema demasiado complejo puede frenar la acción individual, por considerarla insuficiente, o, al contrario, también puede provocar que el público no se lo tome demasiado en serio ante el exceso de proclamas apocalípticas.

Este estudio surgió por el interés de examinar si los contenidos de YouTube sobre cambio climático contribuyen a que se generen nuevas propuestas para la comunicación del tema, de modo que se superen las limitaciones de la representación que llevan a cabo los medios de comunicación convencionales. Sin embargo, los resultados de nuestro estudio indican que la representación del cambio climático en YouTube replica algunas de las características principales de la representación que llevan a cabo los medios convencionales, la cual ha demostrado ser ineficaz para promover suficientemente la implicación de los ciudadanos en la lucha contra este proceso. Esto implica que se están perdiendo oportunidades para representar el cambio climático de un modo que conlleve un mayor potencial para comprometer a la ciudadanía. 


\section{Referencias}

Alexa. (2018). Los mejores 500 sitios en la web. https://www.alexa.com/ topsites

Babbel. (2016). The 10 most spoken languages in the world. https://www. babbel.com/en/magazine/the-10-most-spoken-languages-inthe-world

Barkemeyer, R., Figge, F., Hoepner, A., Holt, D., Kraak, J. M. y Yu, P.S. (2017). Media coverage of climate change: An international comparison. Environment and Planning C: Politics and Space, 35(6), 1029-1054. https://doi.org/10.1177/0263774X16680818

Bauchner, H. y Fontanarosa, P. B. (2014). Climate change: A continuing threat to the health of the world's population. Journal American Medical Association, 312(15). https://doi.org/10.1001/ jama.2014.13094

Boykoff, J. (2012). US media coverage of the Cancun climate change conference. PS: Political Science and Politics, 45(2), 251-258. https:// doi.org/10.1017/S104909651100206X

Boykoff, M. T. y Boykoff,J. M. (2004). Balance as bias: Global warming and the US prestige press. Global Environmental Change, 14(2), 125136. https://doi.org/10.1016/j.gloenvcha.2003.10.001

Boykoff, M. T. y Boykoff, J. M. (2007). Climate change and journalistic norms: A case-study of US mass-media coverage. Geoforum, 38(6), 1190-1204. https://doi.org/10.1016/j.geoforum.2007.01.008

Boykoff, M. T. y Roberts, J. T. (2007). Media coverage of climate change: Current trends, strengths, weaknesses. Human Development Report, 2008(3). http://citeseerx.ist.psu.edu/viewdoc/download? doi $=10.1 .1 .422 .4961 \&$ rep=rep $1 \&$ type $=$ pdf 
Boykoff, M. (2016, 7 de marzo). Why did Paris climate summit get less press coverage than Copenhagen. ClimateChangeNews.com. http:// www.climatechangenews.com/2016/03/07/why-did-paris-climate-summit-get-less-press-coverage-than-copenhagen/

Boykoff, M., Andrews, K., Daly, M., Katzung, J., Luedecke, G., Maldonado, C. y Nacu-Schmidt, A. (2018). A Review of Media Coverage of Climate Change and Global Warming in 2017. https://sciencepolicy. colorado.edu/icecaps/research/media_coverage/summaries/special_issue_2017.htmlhttp://sciencepolicy.colorado.edu/icecaps/ research/media_coverage/summaries/2017_special_issue.pdf

Brulle, R. J., Carmichael, J. y Jenkins, J. C. (2012). Shifting public opinion on climate change: An empirical assessment of factors influencing concern over climate change in the US, 2002-2010. Climatic Change, 114(2), 169-188. https://doi.org/10.1007/s10584-012-0403-y

Cisco. (2017). Cisco Visual Networking Index: Forecast and Methodology, 2016-2021. https://www.cisco.com/c/en/us/solutions/collate$\mathrm{ral} /$ service-provider/visual-networking-index-vni/complete-white-paper-c11-481360.html

Cisco. (2019). VNI Forecast Highlights Tool. https://www.cisco.com/c/m/ en_us/solutions/service-provider/vni-forecast-highlights.html

Dawkins, R. (1994). El gen egoísta: las bases biológicas de nuestra conducta. Salvat.

Ereaut, G. y Segnit, N. (2006, 3 de agosto). Warm words: How we are telling the climate story and can we tell it better. https://www.ippr.org/publications/warm-wordshow-are-we-telling-the-climate-story-andcan-we-tell-it-better

Erviti, M. C. (2013). Las imágenes del cambio climático en los informativos de televisión: análisis de seis televisiones españolas de cobertura nacio- 
nal (Tesis doctoral, Universidad de Navarra). http:// dadun.unav. edu/handle/10171/39019

Erviti, M. C. (2018). Producing science online video. En B. León y M. Bourk (eds.), Communicating science and technology through online video: Researching a new media phenomenon (pp. 28-40). Routledge.

Erviti, M. C. y León, B. (2014). La comunicación de la ciencia a través del vídeo online: contenidos populares en YouTube. En J. Sierra Sánchez y D. Rodrigues Parente (coords.), Contenidos digitales en la era de la sociedad conectada (pp. 129-146). Fragua.

Fernández-Reyes, R. (2016). Contraste entre la cobertura de la Cumbre de Copenhague y la Cumbre de París en prensa española. Redes.com: Revista de Estudios para el Desarrollo Social de la Comunicación, 13, 77-103. https://idus.us.es/handle/11441/55977

Fernández-Reyes, R., Piñuel-Raigada, J.y Vicente-Mariño, M. (2015). La cobertura periodística del cambio climático y del calentamiento global en El País, El Mundo y La Vanguardia. Revista Latina de Comunicación Social, 70, 122-140. https://doi.org/10.4185/RLCS-2015-1038

Gurwitt, S., Malkki, K. y Mitra, M. (2017). Global issue, developed country bias: The Paris climate conference as covered by daily print news organizations in 13 nations. Climatic Change, 143(3-4), 281-296. https://doi.org/10.1007/s10584-017-2004-2

Herring, S. C., Hoerling, M. P., Kossin, J. P., Peterson, T. C. y Stott, P. A. (2015). Explaining extreme events of 2014 from a climate perspective. Bulletin of the American Meteorological Society, 96(12), S1-S172.https://doi.org/10.1175/BAMS-ExplainingExtremeEvents2014.1

Huerta, J. R. (2017, 28 de noviembre). El cambio climático y los desafíos para la salud. http://www.medicosypacientes.com/opinion/dr-joseramon-huerta-el-cambio-climatico-y-los-desafios-para-la-salud 
Informe21.com. (2018, 11 de septiembre). Cambio climático contribuyó al aumento mundial del hambre en 2017. https://informe21.com/ ciencia-y-tecnologia/cambio-climatico-contribuyo-al-aumentomundial-del-hambre-en-2017

León, B. (2013). La representación del cambio climático en los medios españoles. En B. León (ed.), El periodismo ante el cambio climático: nuevas perspectivas $y$ retos (pp. 11-44). UOC.

López-Noguero, F. (2002). El análisis de contenido como método de investigación. Revista de Educación, 4, 167-179. http://rabida.uhu. es/dspace/handle/10272/1912

Maibach, E. W., Nisbet, M., Baldwin, P., Akerlof, K. y Diao, G. (2010). Reframing climate change as a public health issue: An exploratory study of public reactions. BMC Public Health, 10(1), 1-11. https:// doi.org/10.1186/1471-2458-10-299

Mason, P. (2011). Why its still kicking off everywhere: The new global revolutions. Verso.

Meira Cartea, P. Á. (dir.) (2013). La respuesta de la sociedad española ante el cambio climático. Fundación Mapfre.

Nimbus. (2014, 4 de agosto). Luglio 2014 al nord Italia: Frescura non eccezionale, ma piogge straordinarie e pochissimo sole. http://www.nimbus. it/clima/2014/140804Luglio2014.htm

Nisbet, M. C. (2009). Communicating climate change: Why frames matter for public engagement. Environment: Science and policy for sustainable development, 51(2), 12-23. https://doi.org/10.3200/ ENVT.51.2.12-23

Nisbet, M. C. y Scheufele, D. A. (2009). What's next for science communication? Promising directions and lingering distractions. American 
Journal of Botany, 96(10), 1767-1778. https://doi.org/10.3732/ ajb.0900041

NyTimes.com. (2018, 11 de septiembre). Los aeropuertos cerca del nivel del mar también son golpeados por el cambio climático. https:// www.nytimes.com/es/2018/09/11/aeropuerto-osaka-cambioclimatico/

Olesen, T. (2015). Global injustice symbols and social movements. Palgrave.

Pasquaré Mariotto, F. y Venturini, C. (2017). 2014, The "year without a summer" in Italy: News media coverage and implications for the climate change debate. Environment, Development and Sustainability, 19(4), 1367-80. https://doi.org/10.1007/s10668-016-9805-8

Real Academia Española. (2014). Hábitat. En Diccionario de la lengua española (23.a). https://dle.rae.es/hábitat

Salazar, J. M. L.y Barroga-Jamias, S. (2014). YouTube for the environment: Characteristics, themes and communication approaches of Philippine biodiversity videos. Asia Life Sciences, 23(1), 267-84. https:// agris.fao.org/agris-search/search.do?recordID $=$ PH2015000373

Stokes, B., Wike, R. y Carle, J. (2015, 5 de noviembre). Global concern about climate change, broad support for limiting emissions. https://www. pewresearch.org/global/2015/11/05/global-concern-about-climate-change-broad-support-for-limiting-emissions/

Theguardian.com. (2018, 25 de septiembre). Climate gentrification: The rich can afford to move - what about the poor? https://www.theguardian.com/environment/2018/sep/25/climate-gentrificationphoenix-flagstaff-miami-rich-poor

Weingart, P., Engels, A. y Pansegrau, P. (2000). Risks of communication: Discourses on climate change in science, politics, and the mass media. Public Understanding of Science, 9(3), 261-284. https://doi. org/10.1088/0963-6625/9/3/304 
Whitmarsh, L. (2008). Are flood victims more concerned about climate change tan other people? The role of direct experience in risk perception and behavioural response. Journal of Risk Research, 11(3), 351-74. https://doi.org/10.1080/13669870701552235

Wilson, K. (2000). Communicating climate change through the media: Predictions, politics y perceptions of risk. En S. Allan, B. Adam y C. Carter (eds.), Environmental risks y the media (pp. 201-217). Routledge.

YouTube. (2019). Estadísticas. https://www.youtube.com/intl/es/about/ press/

Zenith. (2017). Top 30 global media owners 2017. https://www.zenithusa. com/top-30-global-media-owners-2017/ 